\title{
Effective Nonvanishing of Pluri-adjoint Line Bundles
}

\author{
Tomoki ARAKAWA
}

Sophia University

(Communicated by H. Tahara)

\begin{abstract}
Let $L$ be an ample line bundle over a smooth complex projective $n$-fold $X$ such that $K_{X}+L$ is nef. We prove that $H^{0}\left(X, \mathcal{O}_{X}\left(m\left(K_{X}+L\right)\right)\right) \neq 0$ holds for every $m \geq n(n+1) / 2+2$.
\end{abstract}

\section{Introduction}

Let $X$ be a smooth projective variety over $\mathbf{C}$ and $L$ an ample line bundle over $X$. Then the pair $(X, L)$ is called a polarized manifold.

In the classification theory of polarized manifolds, it is important to study a condition on the integer $m$ for which $\left|K_{X}+m L\right|$ is free. Fujita's freeness conjecture ([6]) predicts that $\left|K_{X}+m L\right|$ is free for any $m \geq \operatorname{dim} X+1$. In [11], Y. Kawamata gave an affirmative answer to this conjecture in the case of $\operatorname{dim} X \leq 4$. In higher dimensional case, H. Tsuji ([17]) proved that $\left|K_{X}+m L\right|$ is free for any $m \geq \operatorname{dim} X(\operatorname{dim} X+1) / 2+1$ (see also [2]).

On the other hand when $K_{X}+L$ is nef, by virtue of the nonvanishing theorem due to V. Shokurov ([16]), $H^{0}\left(X, \mathcal{O}_{X}\left(m\left(K_{X}+L\right)\right)\right) \neq 0$ holds for $m \gg 0$. Then it is important to find an integer $m$ with $H^{0}\left(X, \mathcal{O}_{X}\left(m\left(K_{X}+L\right)\right)\right) \neq 0$. Concerning this, F. Ambro ([1]) and Y. Kawamata ([12]) proposed the following conjecture:

CONJeCtURe 1.1. Let $X$ be a normal projective variety and let $B$ be a $\mathbf{Q}$-effective divisor on $X$ such that $(X, B)$ is a KLT pair. Let $D$ be a nef Cartier divisor on $X$ such that $D-\left(K_{X}+B\right)$ is nef and big. Then $H^{0}\left(X, \mathcal{O}_{X}(D)\right) \neq 0$ holds.

We note that if $X$ is smooth, $B=0$ and $D:=K_{X}+L$ is nef, this implies that $H^{0}\left(X, \mathcal{O}_{X}\left(K_{X}+L\right)\right) \neq 0$ holds for every polarized manifold $(X, L)$ with $K_{X}+L$ nef. In [12], Kawamata solved the conjecture above when $X$ is 2-dimensional and when $X$ is a minimal 3-fold. In [10], A. Hoering solved it when $X$ is a normal projective 3-fold with at most Q-factorial canonical singularities, $B=0$, and $D-K_{X}$ is a nef and big Cartier divisor. These results are immediate consequences of the Hirzebruch-Riemann-Roch theorem and some classical results on surfaces and 3-folds. However in higher dimensional case, it is

Received August 6, 2013; revised October 6, 2014

Mathematics Subject Classification: 14C20 (Primary), 14J40 (Secondary)

Key words and phrases: Polarized manifolds, adjoint bundles, effective nonvanishing 
rather difficult to calculate $\operatorname{dim} H^{0}\left(X, \mathcal{O}_{X}(D)\right)$. In fact, Conjecture 1.1 is still widely open for the case of $\operatorname{dim} X \geq 4$.

Concerning the effective nonvanishing of global sections of pluri-adjoint line bundles, Y. Fukuma ([7]) proposed the following problem:

PRoblem 1.2. Let $(X, L)$ be a polarized manifold of dimension $n$ with $\kappa\left(K_{X}+L\right) \geq 0$. Find a positive integer $m_{n}$ depending only on $n$ such that

$$
H^{0}\left(X, \mathcal{O}_{X}\left(m\left(K_{X}+L\right)\right)\right) \neq 0
$$

holds for every $m \geq m_{n}$.

For the case of $\operatorname{dim} X \leq 4$, several results have been obtained (cf. [7], [8], [10]). In higher dimensional case, the problem is yet to be investigated. In fact the existence of the uniform bound $m_{n}$ as above still remains open.

The purpose of this article is to give the following partial answer to Problem 1.2 in higher dimensional case.

THEOREM 1.3. Let $(X, L)$ be a polarized manifold of dimension $n$ such that $K_{X}+L$ is nef. Then

$$
H^{0}\left(X, \mathcal{O}_{X}\left(m\left(K_{X}+L\right)\right)\right) \neq 0
$$

holds for every positive integer $m \geq n(n+1) / 2+2$.

We prove the theorem above in Section 4. The outline of the proof is as follows: By the nefness of $K_{X}+L$ and the base point free theorem, we obtain a surjective morphism $f: X \longrightarrow Y$ to a smooth projective variety $Y$. Moreover, we obtain a big divisor $B$ on $Y$ such that $K_{Y}+B$ is nef and big (Lemmas 4.2 and 4.3). Then we can reduce the problem to the study of the effective nonvanishing of global sections of multiples of $K_{Y}+B$. Namely, it suffices to show the following:

THEOREM 1.4. Let $Y$ be a smooth projective variety of dimension $d, N$ a nef and big divisor on $Y$ and $B$ a big divisor on $Y$. Then

$$
H^{0}\left(Y, \mathcal{O}_{Y}\left(K_{Y}+m N+B\right)\right) \neq 0
$$

holds for every $m \geq d(d+1) / 2+1$.

Letting $N:=K_{Y}+B$, by the theorem above, we deduce Theorem 1.3. In Section 3, we prove Theorem 1.4, using the Kawamata-Shokurov concentration method ([13, Chapter 2]) and the technique adopted by Angehrn and Siu ([2]), and Tsuji ([17]) in their study of Fujita's freeness conjecture.

Throughout this paper, we work over the field of complex numbers C. Furthermore we use the words line bundle and invertible sheaf interchangeably. If $D$ is a Cartier divisor on a variety $X$, then $\mathcal{O}_{X}(D)$ denotes the corresponding line bundle over $X$. 


\section{Preliminaries}

In this section, we review some algebraic and analytic notions.

2.1. Nef and big line bundles. In this subsection, we shall recall some properties of nef and big line bundles.

Definition 2.1. Let $X$ be a normal variety and $D$ a Cartier divisor on $X$. Then

1. $D$ is said to be nef, if $D \cdot C \geq 0$ holds for every irreducible curve $C$ on $X$.

2. $D$ is said to be big, if $\kappa(X, D)=\operatorname{dim} X$ holds, where $\kappa(X, D)$ denotes the Iitaka-Kodaira dimension of $D$ defined by

$$
\kappa(X, D)=\limsup _{m \rightarrow \infty} \frac{\log \operatorname{dim} H^{0}\left(X, \mathcal{O}_{X}(m D)\right)}{\log m} .
$$

The following proposition called Kodaira's lemma is fundamental.

Proposition 2.2. Let $X$ be a normal complete variety and $D$ a big divisor on $X$. Then for an arbitrary divisor $M$, we have $|m D-M| \neq \emptyset$ for $m \gg 0$.

Proof. See [13, Lemma 0-0-3].

By using Kodaira's lemma and the Riemann-Roch theorem, we have the following:

Proposition 2.3. Let $X$ be a smooth projective variety of dimension $n$ and $D$ a nef and big divisor on $X$. Then

$$
h^{0}\left(X, \mathcal{O}_{X}(m D)\right)=\frac{D^{n}}{n !} m^{n}+O\left(m^{n-1}\right)
$$

holds, where $h^{0}\left(X, \mathcal{O}_{X}(m D)\right):=\operatorname{dim} H^{0}\left(X, \mathcal{O}_{X}(m D)\right)$.

ProOF. See [5, Corollary 4.3].

2.2. Singularities of divisors. In this subsection, we review the notion of singularities of pairs.

Definition 2.4. Let $X$ be a normal variety and $U:=X_{\text {reg }}$ the nonsingular locus of $X$. Since $\operatorname{codim}(X \backslash U) \geq 2$ holds, every divisor on $X$ is uniquely determined by its restriction to $U$ (cf. [9, Chapter II]). Then we can define the canonical sheaf $\omega_{X}=\mathcal{O}_{X}\left(K_{X}\right)$ of $X$ by

$$
\omega_{X}:=i_{*} \mathcal{O}_{U}\left(K_{U}\right),
$$

where $i: U \hookrightarrow X$ is the inclusion.

DEFINITION 2.5. Let $(X, D)$ be a pair of a normal variety $X$ and an effective $\mathbf{Q}$-divisor $D$ on $X$. A $\log$ resolution of $(X, D)$ is a proper birational morphism $\mu: Y \longrightarrow X$ such that $Y$ is smooth and $\operatorname{Exc}(\mu) \cup \mu_{*}^{-1} D$ has a simple normal crossing support, where $\operatorname{Exc}(\mu) \subset X$ denotes the exceptional locus of $\mu$, and $\mu_{*}^{-1} D$ denotes the strict transform of $D$ on $Y$. 
In order to consider singularities of pairs, we shall introduce the following notion.

DEFINITION 2.6. Let $(X, D)$ be a pair of a normal variety and an effective $\mathbf{Q}$-divisor on $X$. Suppose that $K_{X}+D$ is $\mathbf{Q}$-Cartier. Let $\mu: Y \rightarrow X$ be a $\log$ resolution of $(X, D)$. Then we have the formula:

$$
K_{Y}=\mu^{*}\left(K_{X}+D\right)+\sum_{i} a_{i} E_{i}
$$

where $E_{i}$ is a prime divisor and $a_{i} \in \mathbf{Q}$. We call $a_{i}$ the discrepancy coefficient for $E_{i}$. The pair $(X, D)$ is said to have only Kawamata log terminal singularities (KLT) (resp. log canonical singularities (LC)), if $a_{i}>-1$ (resp. $a_{i} \geq-1$ ) holds for every $i$. The pair $(X, D)$ is said to be KLT (resp. LC) at a point $x \in X$, if $\left(U,\left.D\right|_{U}\right)$ is KLT (resp. LC) for some neighborhood $U$ of $x$.

Definition 2.7. Let $(X, D)$ be a pair of a normal variety and an effective $\mathbf{Q}$-divisor on $X$. A subvariety $W$ of $X$ is said to be a center of $\log$ canonical singularities for $(X, D)$, if there are a $\log$ resolution $\mu: Y \longrightarrow X$ and a prime divisor $E$ on $Y$ with the discrepancy coefficient $e \leq-1$ such that $\mu(E)=W$. The set of all centers of log canonical singularities for $(X, D)$ is denoted by $C L C(X, D)$. For a point $x \in X$, we set $C L C(X, x, D):=\{W \in$ $C L C(X, D) \mid x \in W\}$.

PROPOSITION 2.8. Let $(X, D)$ be a pair of a normal variety and an effective $\mathbf{Q}-$ Cartier divisor such that $K_{X}+D$ is $\mathbf{Q}$-Cartier. Assume that $X$ is $K L T$ and $(X, D)$ is LC. If $W_{1}$ and $W_{2}$ are the elements of $C L C(X, D)$ and $W$ is an irreducible component of $W_{1} \cap W_{2}$, then $W \in C L C(X, D)$. This implies that if $(X, D)$ is LC but not KLT at a point $x \in X$, there exists the unique minimal element of $C L C(X, x, D)$. (This minimal element is called the minimal center of log canonical singularities of $(X, D)$ at $x$.)

ProOF. See [11, Proposition 1.5].

2.3. Singular hermitian metrics and multiplier ideal sheaves. In the proof of Theorem 1.3, we use singular hermitian metrics as in [5]. We here review the notions of singular hermitian metrics and multiplier ideal sheaves.

DEFINITION 2.9. Let $L$ be a holomorphic line bundle over a complex manifold $X$. A singular hermitian metric $h$ on $L$ is given by

$$
h=h_{0} \cdot e^{-\varphi},
$$

where $h_{0}$ is a $C^{\infty}$-hermitian metric on $L$ and $\varphi \in L_{\text {loc }}^{1}(X)$ is an $L^{1}$-local function on $X$. Then we call $\varphi$ the weight function of $h$ with respect to $h_{0}$, and $(L, h)$ a singular hermitian line bundle over $X$. The curvature current $\Theta_{h}$ of $h$ is defined by

$$
\Theta_{h}=\Theta_{h_{0}}+\sqrt{-1} \partial \bar{\partial} \varphi,
$$

where $\Theta_{h_{0}}$ denotes the curvature form of $h_{0}$ and $\partial \bar{\partial}$ is taken in the sense of currents. 
EXAMPLE 2.10. Let $L$ be a holomorphic line bundle over a complex manifold $X$. Suppose that there exists a positive integer $m$ with $\Gamma\left(X, L^{\otimes m}\right) \neq 0$. Let $\sigma \in \Gamma\left(X, L^{\otimes m}\right)$ be a nontrivial section. Then

$$
h:=\frac{1}{|\sigma|^{2 / m}}=\frac{h_{0}}{h_{0}^{\otimes m}(\sigma, \sigma)^{1 / m}}
$$

is a singular hermitian metric on $L$, where $h_{0}$ is an arbitrary $C^{\infty}$-hermitian metric on $L$ (the right hand side is independent of $h_{0}$ ). Then by Poincaré-Lelong's formula, the curvature current $\Theta_{h}$ is given by

$$
\Theta_{h}=\frac{2 \pi}{m}(\sigma),
$$

where $(\sigma)$ denotes the current of integration over the divisor of $\sigma$.

DEFINITION 2.11. Let $(L, h)$ be a singular hermitian line bundle over a complex manifold $X$. The $L^{2}$-sheaf $\mathcal{L}^{2}(L, h)$ of $(L, h)$ is defined by

$$
\mathcal{L}^{2}(L, h)(U):=\left\{\sigma \in \Gamma(U, L) \mid h(\sigma, \sigma) \in L_{\mathrm{loc}}^{1}(U)\right\},
$$

where $U$ runs over the open subsets of $X$.

Now we shall write $h$ as $h=h_{0} \cdot e^{-\varphi}$, where $h_{0}$ is a $C^{\infty}$-hermitian metric on $L$ and $\varphi \in L_{\mathrm{loc}}^{1}(X)$ is the weight function of $h$ with respect to $h_{0}$. Then we define the multiplier ideal sheaf $\mathcal{I}(h)$ of $(L, h)$ by

$$
\mathcal{I}(h):=\mathcal{L}^{2}\left(\mathcal{O}_{X}, e^{-\varphi}\right) .
$$

Then we see that $\mathcal{L}^{2}(L, h)=\mathcal{O}_{X}(L) \otimes \mathcal{I}(h)$ holds.

Multiplier ideal sheaves are very useful in investigating singularities of pairs. Using the notation above, we shall define the multiplier ideal sheaves of divisors as follows:

DEFINITION 2.12. Let $X$ be a smooth projective variety and let $D=\sum_{i} a_{i} D_{i}$ be an effective $\mathbf{Q}$-divisor on $X$. For every $i$, let $\sigma_{i} \in \Gamma\left(X, \mathcal{O}_{X}\left(D_{i}\right)\right)$ be a global section with divisor $D_{i}$, and let $h_{i}$ be a $C^{\infty}$-hermitian metric on $\mathcal{O}_{X}\left(D_{i}\right)$. Then we define the multiplier ideal sheaf $\mathcal{I}(D)$ of the divisor $D$ by

$$
\mathcal{I}(D):=\mathcal{I}\left(\frac{1}{\prod_{i} h_{i}\left(\sigma_{i}, \sigma_{i}\right)^{a_{i}}}\right) .
$$

The following proposition reveals a relation between multiplier ideal sheaves and singularities of pairs (cf. Definition 2.6).

Proposition 2.13. Let $X$ be smooth projective variety of dimension $n$ and let $D$ be an effective $\mathbf{Q}$-divisor on $X$. Then $(X, D)$ is KLT at a point $x$ of $X$, if and only if $\mathcal{I}(D)_{x}=$ $\mathcal{O}_{X, x}$ holds. In particular, if the multiplicity of $D$ at $x$ is greater than or equal to $n$, then $\mathcal{I}(D)_{x} \neq \mathcal{O}_{X, x}$. 
PROOF. See [14, Proposition 3.20].

In proving Theorem 1.4, the following vanishing theorem by Nadel ([15]) plays an important role.

THEOREM 2.14. Let $(L, h)$ be a singular hermitian line bundle over a compact Kähler manifold $X$ and $\omega$ be a Kähler form on X. Suppose that the curvature current $\Theta_{h}$ of $h$ is strictly positive, i.e., there exists a positive constant $\varepsilon>0$ such that $\Theta_{h}-\varepsilon \omega$ is a positive $(1,1)$-current. Then $\mathcal{I}(h)$ is a coherent sheaf on $X$, and

$$
H^{q}\left(X, \mathcal{O}_{X}\left(K_{X}+L\right) \otimes \mathcal{I}(h)\right)=0
$$

holds for every $q \geq 1$.

\section{Proof of Theorem 1.4}

In this section we prove Theorem 1.4. We set $\mu_{0}:=N^{d}$ and fix a point $y_{0}$ on $Y$. First we shall construct a singular hemitian metric on some multiple of $N$ with sufficiently large singularity at $y_{0}$.

LEMMA 3.1. Let $\varepsilon$ be a positive number with $\varepsilon<1$. Then

$$
H^{0}\left(Y, \mathcal{O}_{Y}(m N) \otimes \mathfrak{m}_{y_{0}}^{\left\lceil\sqrt[d]{\mu_{0}}(1-\varepsilon) m\right\rceil}\right) \neq 0
$$

holds for any sufficiently large integer $m$.

PROOF. We consider the exact sequence:

$$
\begin{aligned}
0 \longrightarrow \mathcal{O}_{Y}(m N) \otimes \mathfrak{m}_{y_{0}}^{\left\lceil\sqrt[d]{\mu_{0}}(1-\varepsilon) m\right\rceil} & \longrightarrow \mathcal{O}_{Y}(m N) \\
& \longrightarrow \mathcal{O}_{Y}(m N) \otimes \mathcal{O}_{Y} / \mathfrak{m}_{y_{0}}^{\left\lceil\sqrt[d]{\mu_{0}}(1-\varepsilon) m\right\rceil} \longrightarrow 0
\end{aligned}
$$

Since $N$ is nef and big, by Proposition 2.3, we have

$$
h^{0}\left(Y, \mathcal{O}_{Y}(m N)\right)=\frac{\mu_{0}}{d !} m^{d}+O\left(m^{d-1}\right)
$$

for $m \gg 1$.

On the other hand, for a positive integer $k$, the sheaf $\mathcal{O}_{Y} / \mathfrak{m}_{y_{0}}^{k}$ is a skyscraper sheaf at $y_{0}$ and its rank is equal to

$$
\left(\begin{array}{c}
d+k-1 \\
d
\end{array}\right)=\frac{k^{d}}{d !}+O\left(k^{d-1}\right) .
$$

Then letting $k:=\left\lceil\sqrt[d]{\mu_{0}}(1-\varepsilon) m\right\rceil$, by the exact sequence above and (3.1), we obtain the conclusion.

Let us fix a positive number $\varepsilon<1$ and a positive integer $m_{0}$ with

$$
H^{0}\left(Y, \mathcal{O}_{Y}\left(m_{0} N\right) \otimes \mathfrak{m}_{y_{0}}^{\left\lceil\sqrt[d]{\mu_{0}}(1-\varepsilon) m_{0}\right\rceil}\right) \neq 0
$$


as in Lemma 3.1. Then we take a nontrivial global section

$$
\sigma_{0} \in H^{0}\left(Y, \mathcal{O}_{Y}\left(m_{0} N\right) \otimes \mathfrak{m}_{y_{0}}^{\left\lceil\sqrt[d]{\mu_{0}}(1-\varepsilon) m_{0}\right\rceil}\right) .
$$

We define the effective $\mathbf{Q}$-divisor $\Delta_{0}$ on $Y$ by

$$
\Delta_{0}=\frac{1}{m_{0}}\left(\sigma_{0}\right)
$$

and the singular hermitian metric $h_{0}$ on $N$ by

$$
h_{0}=\frac{1}{\left|\sigma_{0}\right|^{2 / m_{0}}} .
$$

Let $\alpha_{0}$ be the positive number defined by $\alpha_{0}:=\inf \left\{\alpha>0 \mid \mathcal{I}\left(h_{0}^{\alpha}\right)_{y_{0}} \neq \mathcal{O}_{Y, y_{0}}\right\}$. Then by Lemma 2.13, we get the inequality:

$$
\alpha_{0} \leq \frac{d}{\sqrt[d]{\mu_{0}}(1-\varepsilon)} .
$$

Fix an arbitrary positive number $\lambda \ll 1 / d$. Since $\mu_{0} \geq 1$ holds, taking $\varepsilon$ sufficiently small, we may assume that $\alpha_{0} \leq d+\lambda$ holds.

Let $Y_{1}$ be the minimal center of $\log$ canonical singularities of $\left(Y, \alpha_{0} \Delta_{0}\right)$ at $y_{0}$, i.e., $Y_{1}$ is the unique minimal element of $C L C\left(Y, y_{0}, \alpha_{0} \Delta_{0}\right)$ (cf. Proposition 2.8). Then one of the following two cases occurs.

1. $\operatorname{dim} Y_{1}=0$, i.e., $Y_{1}=\left\{y_{0}\right\}$ holds.

2. $\operatorname{dim} Y_{1}>0$ holds.

First we consider the case of $\operatorname{dim} Y_{1}=0$. In this case, we can conclude the following:

LEMMA 3.2. If $\operatorname{dim} Y_{1}=0$ holds, then

$$
H^{0}\left(Y, \mathcal{O}_{Y}\left(K_{Y}+m N+B\right)\right) \neq 0
$$

holds for every $m \geq d+1$.

Proof. We fix an integer $m>\alpha_{0}$. Then by $\alpha_{0} \leq d+\lambda$, we have $m \geq d+1$.

Since $N$ is big, by Proposition 2.2, there exists an effective Q-divisor $G$ on $Y$ such that $N-G$ is ample. We may assume that the support of $G$ does not contain $y_{0}$. Let $\delta>0$ be a sufficiently small rational number, and we set $A:=\left(m-\alpha_{0}\right) N-\delta G$. Note that $A$ is ample, since $N$ is nef. We fix a $C^{\infty}$-hermitian metric $h_{A}$ on $A$ with strictly positive curvature. Then we define the singular hermitian metric $h$ on $m N$ by

$$
h:=\frac{h_{0}^{\alpha_{0}} \cdot h_{A}}{\left|\sigma_{G}\right|^{2 \delta}},
$$

where $\sigma_{G}$ is a multivalued holomorphic section of the $\mathbf{Q}$-line bundle $G$ with divisor $G$. Since $h \cdot h_{B}$ has strictly positive curvature current, it follows from Theorem 2.14 that

$$
H^{1}\left(Y, \mathcal{O}_{Y}\left(K_{Y}+m N+B\right) \otimes \mathcal{I}\left(h \cdot h_{B}\right)\right)=0
$$


holds. This implies that the restriction map:

$$
H^{0}\left(Y, \mathcal{O}_{Y}\left(K_{Y}+m N+B\right)\right) \longrightarrow H^{0}\left(Y, \mathcal{O}_{Y}\left(K_{Y}+m N+B\right) \otimes \mathcal{O}_{Y} / \mathcal{I}\left(h \cdot h_{B}\right)\right)
$$

is surjective. Now we may assume that $y_{0}$ is not on the singular locus of $h_{B}$, and hence $\mathcal{O}_{Y} / \mathcal{I}\left(h \cdot h_{B}\right)$ has isolated support at $y_{0}$. Therefore by the surjectivity of (3.2), there exists a global section $\tau \in H^{0}\left(Y, \mathcal{O}_{Y}\left(K_{Y}+m N+B\right)\right.$ with $\tau\left(y_{0}\right) \neq 0$. In particular we obtain $H^{0}\left(Y, \mathcal{O}_{Y}\left(K_{Y}+m N+B\right)\right) \neq 0$. We have thus proved the lemma.

Next we consider the second case: $\operatorname{dim} Y_{1}>0$. In this case, we need to cut down the support of $\mathcal{O}_{Y} / \mathcal{I}\left(h_{0}^{\alpha_{0}}\right)$. Let $Y^{\circ}$ be the set of points $y$ on $Y$ such that $|m N|$ is free at $y$ and $\Phi_{|m N|}$ is an isomorphism on a neighborhood of $y$ for some $m \geq 1$. Let $y_{1}$ be a regular point of $Y_{1}$ with $y_{1} \in Y^{\circ}$, and we set $\mu_{1}:=N^{d_{1}} \cdot Y_{1}$. Then we see that $\mu_{1} \geq 1$ holds. In the same way as the proof of Lemma 3.1, we have the following:

LEMMA 3.3.

$$
\left.H^{0}\left(Y_{1}, \mathcal{O}_{Y_{1}}(m N) \otimes \mathfrak{m}_{y_{1}}^{\left\lceil d_{1}\right.}{ }^{\mu_{1}}(1-\varepsilon) m\right\rceil\right) \neq 0
$$

holds for every positive number $\varepsilon<1$ and every integer $m \gg 1$.

Let $l_{1}$ be an arbitrary positive integer, which will be specified later. Let $\varepsilon^{\prime}<1$ be a sufficiently small positive number and $m_{1}$ a sufficiently large integer with

$$
\left.H^{0}\left(Y_{1}, \mathcal{O}_{Y_{1}}\left(m_{1} l_{1} N\right) \otimes \mathfrak{m}_{y_{1}}^{\left\lceil d_{1}\right.} \sqrt{\mu_{1}}\left(1-\varepsilon^{\prime}\right) m_{1} l_{1}\right\rceil\right) \neq 0
$$

as in Lemma 3.3. Then we take a nontrivial section

$$
\sigma_{1}^{\prime} \in H^{0}\left(Y_{1}, \mathcal{O}_{Y_{1}}\left(m_{1} l_{1} N\right) \otimes \mathfrak{m}_{y_{1}}^{\left\lceil d_{\sqrt{\mu_{1}}}\left(1-\varepsilon^{\prime}\right) m_{1} l_{1}\right\rceil}\right) .
$$

As in the proof of Lemma 3.2, $G$ is the effective $\mathbf{Q}$-divisor on $Y$ such that $N-G$ is ample. Let $k_{1}$ be a positive integer such that $A_{1}:=k_{1}(N-G)$ is Cartier. Then we have the following:

LEMMA 3.4. There exists a global section $\sigma_{1} \in H^{0}\left(Y, \mathcal{O}_{Y}\left(m_{1}\left(l_{1}+k_{1}\right) N\right)\right)$ such that its vanishing order at $y_{1}$ is at least $\left\lceil\sqrt[d_{1}]{\mu_{1}}\left(1-\varepsilon^{\prime}\right) m_{1} l_{1}\right\rceil$, and the divisor $\left(\sigma_{1}\right)$ is smooth on $Y \backslash\left(Y_{1} \cup \operatorname{Supp} G\right)$.

PROOF. By Serre's vanishing theorem, taking $m_{1}$ sufficiently large, we may assume that $H^{1}\left(Y, \mathcal{O}_{Y}\left(m_{1}\left(l_{1} N+A_{1}\right)\right) \otimes \mathcal{I}_{Y_{1}}\right)=0$ holds, where $\mathcal{I}_{Y_{1}}$ denotes the ideal sheaf of $Y_{1}$. We may also assume that $H^{0}\left(Y_{1}, \mathcal{O}_{Y_{1}}\left(m_{1} A_{1}\right)\right) \neq 0$ holds. Then the restriction map:

$$
H^{0}\left(Y, \mathcal{O}_{Y}\left(m_{1}\left(l_{1} N+A_{1}\right)\right)\right) \longrightarrow H^{0}\left(Y_{1}, \mathcal{O}_{Y_{1}}\left(m_{1}\left(l_{1} N+A_{1}\right)\right)\right)
$$

is surjective.

Let $\tau$ be a general section in $H^{0}\left(Y_{1}, \mathcal{O}_{Y_{1}}\left(m_{1} A_{1}\right)\right)$. Then by the surjectivity of (3.3), the section:

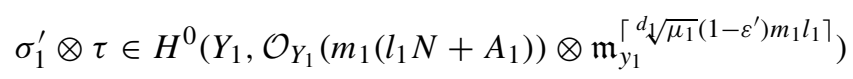


extends to a global section $\sigma_{1} \in H^{0}\left(Y, \mathcal{O}_{Y}\left(m_{1}\left(l_{1} N+A_{1}\right)\right)\right)$. Since $G$ is effective, we may consider $\sigma_{1}$ as an element of $H^{0}\left(Y, \mathcal{O}_{Y}\left(m_{1}\left(l_{1}+k_{1}\right) N\right)\right)$. By virtue of Bertini's theorem and the surjectivity of (3.3), we can take $\sigma_{1}$ such that $\left(\sigma_{1}\right)$ is smooth on $Y \backslash\left(Y_{1} \cup \operatorname{Supp} G\right)$. So we are done.

We define the $\mathbf{Q}$-divisor $\Delta_{1}$ on $Y$ by

$$
\Delta_{1}:=\frac{1}{m_{1}\left(l_{1}+k_{1}\right)}\left(\sigma_{1}\right),
$$

and the singular hermitian metric $h_{1}$ on $N$ by

$$
h_{1}:=\frac{1}{\left|\sigma_{1}\right|^{2 / m_{1}\left(l_{1}+k_{1}\right)}} .
$$

Now let us fix a sufficiently small rational number $0<\varepsilon_{0}<1$. We set

$$
\alpha_{1}:=\inf \left\{\alpha>0 \mid \mathcal{I}\left(h_{0}^{\alpha_{0}-\varepsilon_{0}} \cdot h_{1}^{\alpha}\right)_{y_{1}} \neq \mathcal{O}_{Y, y_{1}}\right\} .
$$

Then we get the following:

LEMMA 3.5. $\alpha_{1} \leq d_{1}+\lambda$ holds, where $\lambda>0$ is the fixed number as mentioned earlier.

For the proof, we use the following elementary lemma (cf. [17, Lemma 6]).

LEMMA 3.6. Let $a, b$ be positive numbers. Then

$$
\int_{0}^{1} \frac{r_{2}^{2 d_{1}-1}}{\left(r_{1}^{2}+r_{2}^{2 a}\right)^{b}} d r_{2}=r_{1}^{2\left(\frac{d_{1}}{a}-b\right)} \int_{0}^{r_{1}^{-a}} \frac{r_{3}^{2 d_{1}-1}}{\left(1+r_{3}^{2 a}\right)^{b}} d r_{3}
$$

holds, where $r_{3}:=r_{2} \cdot r_{1}^{-1 / a}$.

PROOF OF LEMMA 3.5. Let $\left(z_{1}, \ldots, z_{d}\right)$ be a local coordinate on a neighbourhood $U$ of $y_{1}$ in $Y$ with $Y_{1} \cap U=\left\{q \in U \mid z_{d_{1}+1}(q)=\cdots=z_{d}(q)=0\right\}$. Then we set $r_{1}:=\left(\sum_{i=d_{1}+1}^{d}\left|z_{i}\right|^{2}\right)^{1 / 2}$ and $r_{2}:=\left(\sum_{i=1}^{d_{1}}\left|z_{i}\right|^{2}\right)^{1 / 2}$. Now we fix a $C^{\infty}$-hermitian metric $h_{N}$ on $N$ and set $a:=\left\lceil\sqrt[d_{1}]{\mu_{1}}(1-\varepsilon) m_{1} l_{1}\right\rceil$. By the construction of $\sigma_{1}$, there exists a positive constant $C$ such that $\left|\sigma_{1}\right|^{2} \leq C\left(r_{1}^{2}+r_{2}^{2 a}\right)$ holds on a neighbourhood of $y_{1}$. Here $|\cdot|$ denotes the norm with respect to $h_{N}^{m_{1}\left(l_{1}+k_{1}\right)}$.

On the other hand, there exists a positive integer $M$ such that $\left|\sigma_{0}\right|^{-2}=O\left(r_{1}^{-M}\right)$ holds on a neighbourhood of the generic point of $Y_{1} \cap U$, where $|\cdot|$ denotes the norm with respect to $h_{N}^{m_{0}}$.

Then by the definition of $\alpha_{1}$ and Lemma 3.6, we have the inequality:

$$
\alpha_{1} \leq \frac{l_{1}+k_{1}}{l_{1}} \cdot \frac{d_{1}}{\sqrt[d_{1}]{\mu_{1}}\left(1-\varepsilon^{\prime}\right)}+\frac{m_{1}\left(l_{1}+k_{1}\right)}{m_{0}} M \varepsilon_{0} .
$$

Taking $l_{1}$ sufficiently large and taking $\varepsilon^{\prime}$ and $\varepsilon_{0}$ sufficiently small, we obtain the desired inequality. 
Let $Y_{2}$ denote the minimal center of LC singularities of $\left(Y,\left(\alpha_{0}-\varepsilon_{0}\right) \Delta_{0}+\alpha_{1} \Delta_{1}\right)$ at $y_{1}$. Since $\left(Y,\left(\alpha_{0}-\varepsilon_{0}\right) \Delta_{0}\right)$ is KLT and $\left(\sigma_{1}\right)$ is smooth on $Y \backslash\left(Y_{1} \cup \operatorname{Supp} G\right)$, we see that $Y_{2}$ is a proper subvariety of $Y_{1}$.

Repeating the same process, we finally obtain the strictly decreasing sequence of subvarieties of $Y$ :

$$
Y \supsetneq Y_{1} \supsetneq \cdots \supsetneq Y_{r} \supsetneq Y_{r+1}=\left\{y_{r}\right\}
$$

points on $Y$ :

$$
y_{0}, y_{1}, \ldots, y_{r}
$$

where $y_{i}$ is a regular point on $Y_{i}$ with $y_{i} \in Y^{\circ}$ for each $i$, and positive numbers:

$$
\alpha_{0}, \alpha_{1}, \ldots, \alpha_{r}
$$

with the estimates $\alpha_{i} \leq d_{i}+\lambda$, where $d_{i}:=\operatorname{dim} Y_{i}$. Furthermore, we obtain global sections:

$$
\left.\sigma_{0} \in H^{0}\left(Y, \mathcal{O}_{Y}\left(m_{0} N\right)\right), \quad \sigma_{i} \in H^{0}\left(Y, \mathcal{O}_{Y}\left(m_{i}\left(l_{i}+k_{1}\right) N\right)\right)\right) \quad(i=1, \ldots, r)
$$

for some positive integers $m_{0}, \ldots, m_{r}$ and $l_{1}, \ldots, l_{r}$.

Then for $2 \leq i \leq r$, let $\Delta_{i}$ be the effective $\mathbf{Q}$-divisor defined by

$$
\Delta_{i}:=\frac{1}{m_{i}\left(l_{i}+k_{1}\right)}\left(\sigma_{i}\right)
$$

and $h_{i}$ the singular hermitian metric on $N$ defined by

$$
h_{i}:=\frac{1}{\left|\sigma_{i}\right|^{2 / m_{i}\left(l_{i}+k_{1}\right)}} .
$$

We note that $Y_{j}$ is the minimal center of $\log$ canonical singularities of the pair $\left(Y,\left(\sum_{i=0}^{j-2}\left(\alpha_{i}-\right.\right.\right.$ $\left.\left.\varepsilon_{i}\right) \Delta_{i}+\alpha_{j-1} \Delta_{j-1}\right)$ at $y_{j-1}$ for every $j=2, \ldots, r+1$. Then we can complete the proof by an argument similar to that in the proof of Lemma 3.2 as follows.

Let $m$ be a positive integer with $m>\sum_{i=0}^{r-1}\left(\alpha_{i}-\varepsilon_{i}\right)+\alpha_{r}$. Since $\alpha_{i} \leq d_{i}+\lambda$ holds for every $i$, we have $m \geq d(d+1) / 2+1$. Then we define the singular hermitian metric $h_{+}$on $\left(\sum_{i=0}^{r-1}\left(\alpha_{i}-\varepsilon_{i}\right)+\alpha_{r}\right) N$ by

$$
h_{+}:=\left(\prod_{i=0}^{r-1} h_{i}^{\alpha_{i}-\varepsilon_{i}}\right) \cdot h_{r}^{\alpha_{r}} .
$$

By the construction of $h_{i}$, we see that $\mathcal{O}_{Y} / \mathcal{I}\left(h_{+}\right)$has isolated support at $y_{r}$.

Let us fix a sufficiently small rational number $\delta>0$. Let $A^{\prime}$ be the $\mathbf{Q}$-divisor on $Y$ defined by $A^{\prime}:=\left(m-\sum_{i=0}^{r-1}\left(\alpha_{i}-\varepsilon_{i}\right)-\alpha_{r}\right) N-\delta G$. Since $A^{\prime}$ is ample, there exists a $C^{\infty}$. hermitian metric $h_{A^{\prime}}$ on $A^{\prime}$ with strictly positive curvature. Let $h^{\prime}$ be the singular hermitian 
metric on $m N$ defined by

$$
h^{\prime}:=\frac{h_{+} \cdot h_{A^{\prime}}}{\left|\sigma_{G}\right|^{2 \delta}}
$$

where $\sigma_{G}$ is a multivalued holomorphic section of $G$ with divisor $G$. Taking $y_{0}$ generic, we may assume that $y_{r}$ is not contained in the support of $G$. Hence we see that $h^{\prime}$ has strictly positive curvature current and the support of $\mathcal{O}_{Y} / \mathcal{I}\left(h^{\prime}\right)$ is isolated at $y_{r}$. Then by Theorem 2.14, we have $H^{1}\left(Y, \mathcal{O}_{Y}\left(K_{Y}+m N+B\right) \otimes \mathcal{I}\left(h^{\prime} \cdot h_{B}\right)\right)=0$, and so the restriction map:

$$
H^{0}\left(Y, \mathcal{O}_{Y}\left(K_{Y}+m N+B\right)\right) \longrightarrow H^{0}\left(Y, \mathcal{O}_{Y}\left(K_{Y}+m N+B\right) \otimes \mathcal{O}_{Y} / \mathcal{I}\left(h^{\prime} \cdot h_{B}\right)\right)
$$

is surjective. Now we may assume that $y_{r}$ is not on the singular locus of $h_{B}$, and hence $\mathcal{O}_{Y} / \mathcal{I}\left(h^{\prime} \cdot h_{B}\right)$ has isolated support at $y_{r}$. Thus there exists a global section $\tau^{\prime} \in$ $H^{0}\left(Y, \mathcal{O}_{Y}\left(K_{Y}+m N+B\right)\right)$ with $\tau^{\prime}\left(y_{r}\right) \neq 0$. This completes the proof of Theorem 1.4.

\section{Proof of Theorem 1.3}

We shall prove Theorem 1.3. Let $X$ be a smooth projective variety of dimension $n$ and $L$ an ample line bundle over $X$ such that $K_{X}+L$ is nef. By [16, Theorem 0.2], we have $\kappa\left(X, K_{X}+L\right) \geq 0$. If $\kappa\left(X, K_{X}+L\right)=0$ holds, using [3, Lemma 3.3.2], we have $\mathcal{O}_{X}\left(K_{X}+L\right)=\mathcal{O}_{X}$, and so $H^{0}\left(X, \mathcal{O}_{X}\left(K_{X}+L\right)\right) \cong \mathbf{C}$. Hence it suffices to consider the case of $\kappa\left(X, K_{X}+L\right)>0$.

Since $K_{X}+L$ is nef, we see that $(m-1)\left(K_{X}+L\right)+L$ is ample for every $m \geq 1$. Then by the base point free theorem ([13, Theorem 3-1-1]), we have the following:

LEMMA 4.1. The complete linear system $\left|m\left(K_{X}+L\right)\right|$ is base point free for every positive integer $m \gg 1$. Moreover, there exists a $C^{\infty}$-hermitian metric $h_{0}$ on $K_{X}+L$ with semipositive curvature.

Let $\Phi_{m}: X \rightarrow \mathbf{P} H^{0}\left(X, \mathcal{O}_{X}\left(m\left(K_{X}+L\right)\right)\right)^{*}$ denote the rational map associated with $\left|m\left(K_{X}+L\right)\right|$, where $m$ is a positive integer with $\left|m\left(K_{X}+L\right)\right| \neq \emptyset$. Taking a sufficiently large integer $a$, by Lemma 4.1 , we obtain a surjective morphism $f:=\Phi_{a}: X \longrightarrow Y$, where $Y$ denotes the image of $X$. Further we may assume that $\kappa\left(X, K_{X}+L\right)=\operatorname{dim} Y$ holds, and that $\kappa\left(F, K_{F}+\left.L\right|_{F}\right)=0$ holds for a general fiber $F$ of $f$. Taking a suitable modification, we may also suppose that $Y$ is smooth. Then again by [3, Lemma 3.3.2], we see that $K_{F}+\left.L\right|_{F}$ is linearly equivalent to $\mathcal{O}_{F}$.

Let $l$ be a non-negative integer. We define the reflexive sheaf $B_{l}$ on $Y$ by

$$
B_{l}:=f_{*} \mathcal{O}_{X}\left(K_{X / Y}+L+l\left(K_{X}+L\right)\right)^{* *},
$$

where $K_{X / Y}:=K_{X}-f^{*} K_{Y}$ is the relative canonical sheaf and $* *$ denotes the double dual. Since $K_{F}+\left.L\right|_{F}$ is trivial, $B_{l}$ is an invertible sheaf on $Y$ for every $l \geq 0$. Moreover we have the following:

LEMMA 4.2. $B_{l}$ is big for every $l \geq 0$. 
Proof. Let $h_{L}$ be a $C^{\infty}$-hermitian metric on $L$ with strictly positive curvature and $h_{0}$ a $C^{\infty}$-hermitian metric on $K_{X}+L$ as in Corollary 4.1. Then we define the singular hermitian metric $h_{B_{l}}$ on $B_{l}$ by

$$
h_{B_{l}}(\sigma, \sigma):=\int_{X / Y} h_{0}^{l} \cdot h_{L} \cdot \sigma \wedge \bar{\sigma},
$$

where $\sigma \in \Gamma\left(Y, B_{l}\right)$ is a global section of $B_{l}$. Here the fiber integral in the right hand side is defined by the following property:

$$
\int_{U}\left(\int_{X / Y} h_{0}^{l} \cdot h_{L} \cdot \sigma \wedge \bar{\sigma}\right)=\int_{f^{-1}(U)} h_{0}^{l} \cdot h_{L} \cdot \sigma \wedge \bar{\sigma}
$$

holds for any open set $U$ in $Y$. We note that $h_{B_{l}}$ is smooth on the smooth locus of $f$, and may have singularities on the discriminant locus of $f$. Then by [18, Theorem 1.5] (see also [4, Theorem 0.1]), we see that $h_{B_{l}}$ has strictly positive curvature current. This completes the proof of Lemma 4.2.

We set $B:=B_{0}=f_{*} \mathcal{O}_{X}\left(K_{X / Y}+L\right)^{* *}$. Then we obtain the Q-linear equivalence:

$$
K_{Y}+B \sim \mathbf{Q} \frac{1}{l}\left(K_{Y}+B_{l-1}\right),
$$

and $f_{*} \mathcal{O}_{X}\left(l\left(K_{X}+L\right)\right)^{* *} \cong \mathcal{O}_{Y}\left(K_{Y}+B_{l-1}\right)$ for every $l \geq 1$. Consequently, we have

$$
\begin{aligned}
H^{0}\left(X, \mathcal{O}_{X}\left(m\left(K_{X}+L\right)\right)\right) & \cong H^{0}\left(Y, f_{*} \mathcal{O}_{X}\left(m\left(K_{X}+L\right)\right)^{* *}\right) \\
& \cong H^{0}\left(Y, \mathcal{O}_{Y}\left(m\left(K_{Y}+B\right)\right)\right)
\end{aligned}
$$

for every $m \geq 1$. In order to apply Theorem 1.4, we need to show the following:

LEMMA 4.3. $K_{Y}+B$ is nef and big.

PROOF. It follows immediately from (4.3) that $K_{Y}+B$ is big.

Let $d V_{Y}$ be a $C^{\infty}$-volume form on $Y$. Then we may consider $d V_{Y}^{-1}$ as a $C^{\infty}$-hermitian metric on $K_{Y}$. For every $l \geq 1$, let $h_{l}$ be the singular hermitian metric on $(1 / l)\left(K_{Y}+B_{l-1}\right)$ defined by $h_{l}:=\left(d V_{Y}^{-1} \cdot h_{B_{l-1}}\right)^{1 / l}$. By (4.2), we may regard $h_{l}$ as a singular hermitian metric on $K_{Y}+B$.

Since $h_{B_{l-1}}$ has semipositive curvature current, we see that

$$
\Theta_{h_{l}}=\frac{1}{l} \Theta_{h_{B_{l-1}}}+\frac{1}{l} \Theta_{d V_{Y}^{-1}} \geq \frac{1}{l} \Theta_{d V_{Y}^{-1}}
$$

holds for every $l \geq 1$. Let

$$
\Theta_{h_{B_{l-1}}}=\left(\Theta_{h_{B_{l-1}}}\right)_{\mathrm{ac}}+\left(\Theta_{h_{B_{l-1}}}\right)_{\operatorname{sing}}
$$

be the Lebesgue decomposition, where $\left(\Theta_{h_{B_{l-1}}}\right)_{\text {ac }}$ denotes the absolutely continuous part and $\left(\Theta_{h_{B_{l-1}}}\right)_{\text {sing }}$ denotes the singular part. Then by (4.1), we see that $\left(\Theta_{h_{B_{l-1}}}\right)_{\text {sing }}$ is independent 
of $l$. Hence by taking a suitable modification of $Y$, we may assume that $\left(\Theta_{h_{B_{l-1}}}\right)_{\operatorname{sing}}$ is an effective divisor independent of $l$. Then by the inequality (4.4), we have

$$
\begin{aligned}
\left(K_{Y}+B\right) \cdot C & =\frac{1}{l} \int_{C}\left\{\left(\Theta_{h_{B_{l-1}}}\right)_{\mathrm{ac}}+\Theta_{d V_{Y}^{-1}}\right\}+\frac{1}{l}\left(\Theta_{h_{B_{l-1}}}\right)_{\operatorname{sing}} \cdot C \\
& \geq \frac{1}{l} K_{Y} \cdot C+\frac{1}{l}\left(\Theta_{h_{B_{l-1}}}\right)_{\operatorname{sing}} \cdot C
\end{aligned}
$$

for an irreducible curve $C$. Letting $l$ tend to infinity, we can conclude that $K_{Y}+B$ is nef after taking a suitable modification of $Y$. By the functoricality, we see that $K_{Y}+B$ is nef, without taking such a modification. We have thus proved Lemma 4.3.

Then applying Theorem 1.4 for $N=K_{Y}+B$, we conclude the following:

LEMMA 4.4.

$$
H^{0}\left(Y, \mathcal{O}_{Y}\left(m\left(K_{Y}+B\right)\right)\right) \neq 0
$$

holds for every $m \geq d(d+1) / 2+2$.

By (4.3) and the lemma above, we have completed the proof of Theorem 1.3.

ACKnowledgment. I would like to express my thanks to Professor Hajime Tsuji for his suggestions and constant support.

\section{References}

[1] F. Ambro, Ladders on Fano varieties, Algebraic geometry, 9. J. Math. Sci. (New York), 94 (1999), no. 1, 1126-1135.

[ 2 ] U. Angehrn and Y.-T. SiU, Effective freeness and point separation for adjoint bundles, Invent. Math. 122 (1995), 291-308.

[ 3 ] M. C. Beltrametti and A. J. Sommese, The adjunction theory of complex projective varieties, de Gruyter Expositions in Math. 16 Walter de Gruyter, Berlin, New York, (1995).

[4] B. Berndtsson and M. PAun, Bergman kernels and the pseudoeffectivity of relative canonical bundles, Duke Math. J. 145 (2008), no. 2, 341-378.

[ 5 ] J.-P. Demailly, Singular hermitian metrics on positive line bundles, Proc. Conf. Complex algebraic varieties (Bayreuth, April 26, 1990), edited by K. Hulek, T. Peternell, M. Schneider, F. Schreyer, Lecture Notes in Math., Vol. 1507, Springer-Verlag, Berlin, 1992.

[ 6 ] T. FuJitA, On polarized manifolds whose adjoint bundles are not semipositive, Algebraic Geometry, Sendai, 1985, Advanced Studies Pure Math., Vol. 10, North-Holland, Amsterdam (1987), 167-178.

[ 7 ] Y. FUKUMA, Effective non-vanishing of global sections of multiple adjoint bundles for polarized 3-folds, J. Pure Appl. Algebra 215 (2011), 168-184.

[ 8 ] Y. FUKUMA, Effective non-vanishing of global sections of multiple adjoint bundles for polarized 4-folds, J. Pure Appl. Algebra 217 (2013), 1535-1547.

[ 9 ] R. HARTShorne, Algebraic Geometry, Springer-Verlag (1977).

[10] A. HöRING, On a conjecture of Beltrametti and Sommese, Journal of Algebraic Geometry 21 (2012), 721751.

[11] Y. KaWAmata, Fujita's freeness conjecture for 3-folds and 4-folds, Math. Ann. 308 (1997), 491-505. 
[12] Y. KAWAmata, On effective nonvanishing and base point freeness, Kodaira's issue, Asian J. Math. 4 (2000), 173-181.

[13] Y. Kawamata, K. Matsuda and K. Matsuki, Introduction to the minimal model problem, Adv. St. Pure Math. 10 (1987), 283-360.

[14] J. Kollár, Singularities of pairs, Algebraic geometry-Santa Cruz 1995, Proc. Sympos. Pure Math., vol. 62, Amer. Math. Soc., Providence, RI, 1997, 221-287.

[15] A. M. NADEL, Multiplier ideal sheaves and existence of Kähler-Einstein metrics of positive scalar curvature, Ann. of Math. 132 (1990), 549-596.

[16] V. V. ShokURov, Theorems on non-vanishing, Math. USSR Izv. 26 (1986), 591-604.

[17] H. Tsuji, Global generation of adjoint line bundles, Nagoya Math. J. 142 (1996), 5-16.

[18] H. TsuJI, Variation of Bergman kernels of adjoint line bundles, math.CV/0511342 (2005).

Present Address:

Department of Mathematics,

SOPHIA UNIVERSITY,

KIOICHO, CHIYODA-KU, TOKYO 102-8554, JAPAN.

e-mail: tomoki-a@sophia.ac.jp 